\title{
The high-field magnetic white dwarf LP790-29: Not a fast rotator ${ }^{\star}$
}

\author{
K. Beuermann and K. Reinsch \\ Universitäts-Sternwarte, Geismarlandstr. 11, 37083 Göttingen, Germany \\ Received 21 September 2001 / Accepted 18 October 2001

\begin{abstract}
We have investigated the nature of the magnetic white dwarf LP 790-29 = LHS 2293 by polarimetric monitoring, searching for short-term variability. No periodicity was found and we can exclude rotation periods between $4 \mathrm{~s}$ and 1.5 hour with a high confidence. Maximum amplitudes of sinusoidal variations are $\Delta R<0.009 \mathrm{mag}$ and $\Delta V_{R}<0.7 \%$ for a mean value of the $R$-band circular polarization of $V_{R}=+9.1 \pm 0.3 \%$. Combined with earlier results by other authors, our observation suggests that LP 790-29 is, in fact, an extremely slowly rotating single white dwarf and not an unrecognized fast rotator and/or disguised cataclysmic variable.
\end{abstract}

Key words. stars: white dwarfs - stars: rotation - stars: individual: LP790-29

\section{Introduction}

The large majority of white dwarfs are slow rotators with equatorial velocities $v_{\text {rot }} \sin i<15 \mathrm{~km} \mathrm{~s}^{-1}$ and rotational periods $P_{\text {rot }} \gtrsim 1$ hr (Heber et al. 1997; Koester et al. 1998). Much slower rotation is not detectable in non-magnetic white dwarfs, but easily measureable in magnetic ones by polarimetric monitoring (Schmidt \& Norsworthy 1991; Berdyugin \& Piirola 1999).

Among the magnetic white dwarfs, there is a surprising dichotomy in the distribution of $P_{\text {rot }}$ for magnetic white dwarfs with all stars having either $P_{\text {rot }}<20 \mathrm{~d}$ or $P_{\text {rot }}>100$ yrs (Schmidt \& Norsworthy 1991). Some magnetic white dwarfs rotate surprisingly fast while others are apparently extremely slow. Among the fast ones are the DA white dwarf RE0317-853 with $B \simeq 500 \mathrm{MG}, T_{\text {eff }} \simeq$ $40000 \mathrm{~K}$, and $P_{\text {rot }}=12 \mathrm{~min}$ (Barstow et al. 1995; Ferrario et al. 1997; Burleigh et al. 1999), the DA star PG1015+014 with $B \simeq 120 \mathrm{MG}, T_{\text {eff }} \simeq 14000 \mathrm{~K}$, and $P_{\text {rot }}=99 \mathrm{~min}$ (Wickramasinge \& Cropper 1988; Schmidt \& Norsworthy 1991), and the DAB white dwarf Feige 7 with $B \sim 35 \mathrm{MG}$, $T_{\text {eff }} \simeq 20000 \mathrm{~K}, P_{\text {rot }}=2.2 \mathrm{hr}$ (Liebert et al. 1977 ; Achilleos et al. 1992). Five systems seem to be very slow rotators with $P_{\text {rot }}>100$ yr (Schmidt \& Norsworthy 1991), among them the proven systems, GD229, G240-72 (Berdyugin \& Piirola 1999), and Grw $+70^{\circ} 8247$ (Friedrich \& Jordan 2001), as well as a suspected one, LP790-29 (Liebert et al. 1978).

Send offprint requests to: K. Beuermann, e-mail: beuermann@uni-sw.gwdg.de

* Based on observations collected at the European Southern Observatory, Chile, under programme ID 64.H-0311.
Slow rotation may be caused by coupling of angular momentum into the giant envelope of the progenitor star or the interstellar medium during later stages (Schmidt \& Norsworthy 1991). Fast rotation may be achieved in a double degenerate which ends as a merger (e.g. Segretain et al. 1997) or in a magnetic cataclysmic variable which loses synchronism (Meyer \& Meyer-Hofmeister 1999). If the donor in a mass-transfer binary is nearly substellar and hydrogen rich (short-period AM Herculis binary), the white dwarf is expected to be of spectral type DA which is not the case for LP790-29. Cataclysmic variables with a (partially) degenerate low-mass donor (AM CVn binaries) transfer helium or carbon and typically end as $\mathrm{CO}$ white dwarfs, possibly with a substellar companion (Iben \& Tutukov 1991). The hot white dwarf RE0317-853 has been suggested to be the result of a merger (Barstow et al. 1995; Ferrario et al. 1997) or a mass-transfer binary (Meyer \& Meyer-Hofmeister 1999). The former appears more likely because the primary in RE0317-853 is hot with $T_{\text {eff }} \simeq 40000 \mathrm{~K}$ and all white dwarfs in short-period cataclysmic variables are cool with $T_{\text {eff }} \simeq 9000-15000 \mathrm{~K}$ (Gänsicke 2000). Although definite conclusions in any individual case may be problematic, the detection of rapid rotation of magnetic white dwarfs would clearly help to elucidate their evolutionary history. It also helps to understand the physical processes by which angular momentum is coupled into the environment of the star (Schmidt \& Norsworthy 1991) and may help to define sources of gravitational wave radiation (Heyl 2000).

LP 790-29 = LHS 2293 was discovered by Liebert et al. (1978) and found to be a highly circularly polarized cool white dwarf which shows the Zeeman shifted $\mathrm{C}_{2}$ Swan 


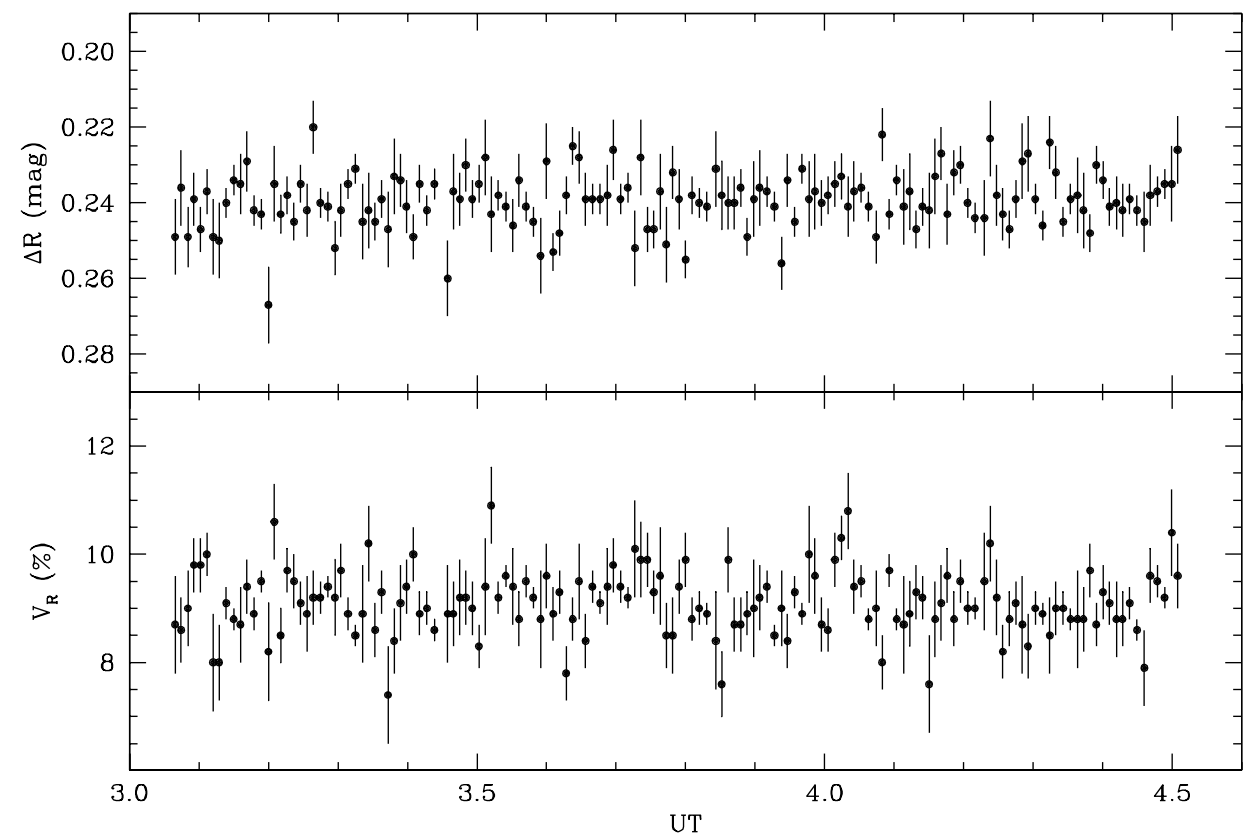

Fig. 1. Time series of the Stokes intensity $I_{R}$ and circular polarization $V_{R}$ in the Bessell $R$-band. The time resolution is $\sim 30-40 \mathrm{~s}$ with 150 individual CCD images taken over $1.5 \mathrm{~h}$. The $R$-band magnitude was measured relative to two comparison stars in the field of LP 790-29.

bands. It has Stokes $V \simeq+8 \%$ and $+9 \%$ at wavelengths shortward of $4300 \AA$ and longward of $5500 \AA$, respectively, (Liebert et al. 1978; West 1989) which decreases to nil inbetween and to $2 \%$ in the $J$-band. Bues (1999) refined its temperature to $T_{\text {eff }} \simeq 7800 \mathrm{~K}$. The field strength was originally quoted as $B \sim 200 \mathrm{MG}$ (Liebert et al. 1978; Schmidt \& Smith 1995), while Bues used 50 MG in her spectral fitting, and Wickramasinghe \& Ferrario (2000) quoted an uncertain $100 \mathrm{MG}$. LP 790-29 is also linearly polarized at the level of $\sim 1 \%$ (West 1989). Polarimetric observations (Liebert et al. 1978; Robert \& Moffat 1989; West 1989) have shown that the level of circular polarization has stayed constant over 10 years, excluding rotation periods in the range of $\sim 20 \mathrm{~min} \lesssim P_{\text {rot }} \lesssim 100 \mathrm{yrs}$ (Schmidt $\&$ Norsworthy 1991). In a non-axisymmetric field geometry, the circular polarization will depend on rotation phase and a short rotation period should be readily detectable in time series of the circular polarization, provided the magnetic axis is inclined for more than a few degrees against the rotational axis and the latter does not point directly at the observer. In this communication, we report results of a search for rapid rotation in LP 790-29, using photometric and polarimetric data taken in the Bessell $R$-band. Given the observed spectral dependence of Stokes $V$ (Liebert et al. 1978; Schmidt et al. 1995), the $R$-band provides the best polarimetric signal of the standard photometric bands.

\section{Observations and results}

We observed LP 790-29 on February 4, 2000, with the ESO $3.6 \mathrm{~m}$ telescope equipped with the focal-reducer spectrograph and camera, EFOSC2, and a user-supplied superachromatic quarter-wave plate which was produced by Halle/Berlin and is of the same type as used in the ESO VLT FORS1 spectrograph. Flux and circular polarization were measured in the photometric Bessell $R$-band with a time resolution of $\sim 30-40 \mathrm{~s}$ for a total of $1.5 \mathrm{~h}$. Pairs of images with the retarder-plate position angle alternating between $-45^{\circ}$ and $+45^{\circ}$ were taken with exposure times of $2 \mathrm{~s}, 3 \mathrm{~s}, 5 \mathrm{~s}$, and $10 \mathrm{~s}$ chosen in random order. The dead time between two exposures was 28-32 s due to readout and instrument-setup times. Reading out single exposures guaranteed the best possible $S / N$ ratio for Stokes $V_{R}$. The $R$-band was chosen because of the higher level of circular polarization compared with that at the shorter wavelengths (Liebert et al. 1978). The measured values of $V_{R}$ have been corrected for instrumental biases and linear-polarization cross-talk, $-1.3 \pm 0.3 \%$, which was determined to the first order from each pair of observations. The error in $V_{R}$ is dominated by this systematic uncertainty, its statistical error is $<0.1 \%$.

Figure 1 shows the resulting time series. The $R$-band magnitude was measured against two comparison stars with similar brightness, USNO 0675_11099946 and USNO 0675_11100282, located $14 \operatorname{arcsec}$ SW and $34 \operatorname{arcsec}$ NE of LP 790-29. In order to search for periodicities, we computed the Analysis of Variance statistics implemented in the European Southern Observatory Munich Image Data Analysis System (MIDAS) software package (Schwarzenberg-Czerny 1989) for periods between the Nyquist limit of $P_{\text {rot }}=4 \mathrm{~s}$ and a maximum period $P_{\text {rot }}=1.5 \mathrm{~h}$ (Fig. 2). The expected value of this statistic for pure noise is $\Theta_{\mathrm{AoV}}=1$. The critical value of the Fisher-Snedecor distribution for a 3- $\sigma$ detection of a periodic signal in the data is $\Theta_{\text {cr }}=2.96$ for 9 and 140 degrees 


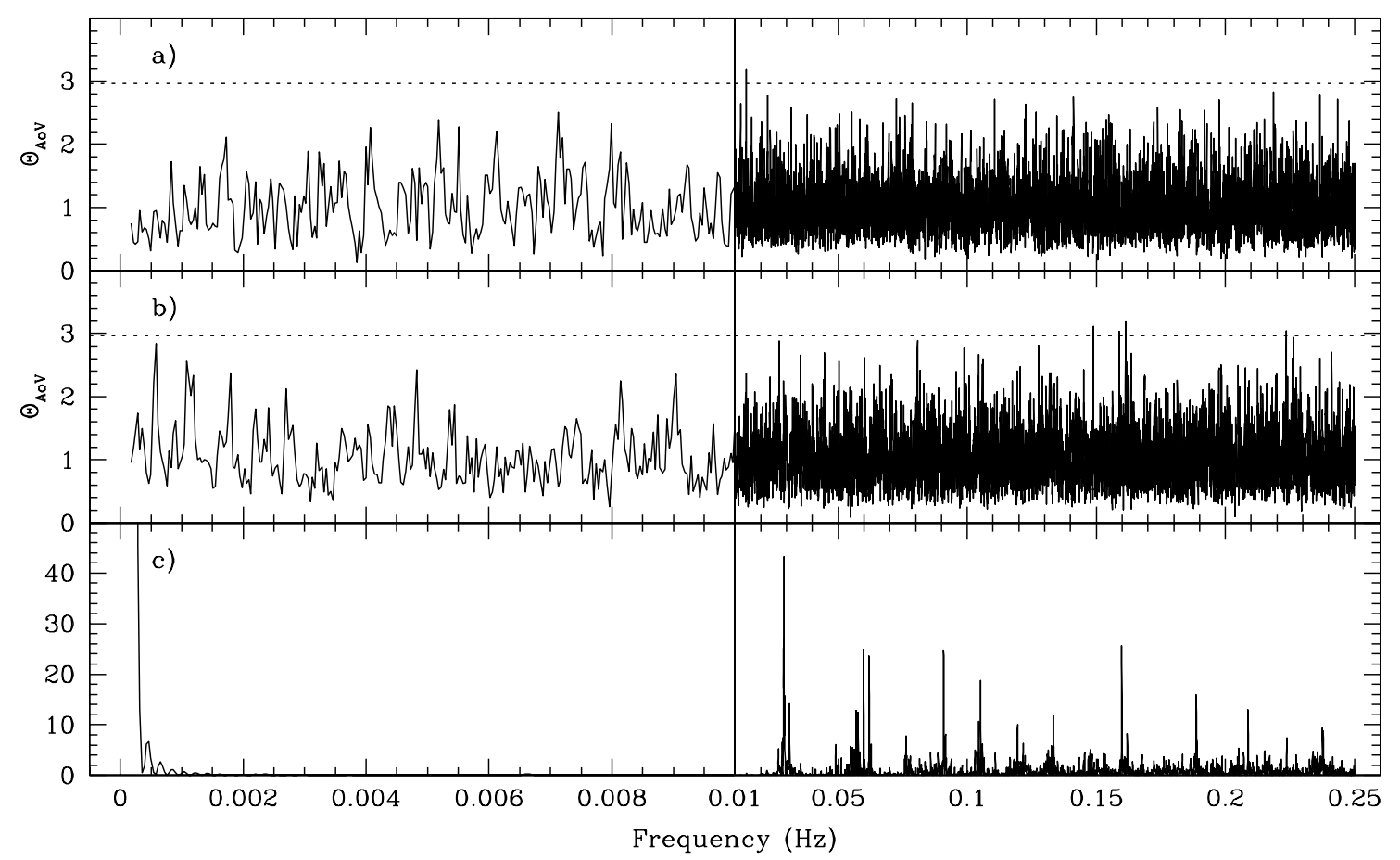

Fig. 2. Periodogram of a) the Stokes intensity $I_{R}$ and b) the circular polarization $V_{R}$ in the Bessell $R$-band, along with c) the spectral window function of the data. The short-dashed lines in panels a) and $\mathbf{b}$ ) give the Fisher-Snedecor critical value for a $3-\sigma$ detection of a periodic signal in the data, uncorrected for bandwidth (see text). The right hand panel covers periods from $4 \mathrm{~s}$ to $100 \mathrm{~s}$, the left hand panel provides an expanded view for periods from $100 \mathrm{~s}$ to $1.5 \mathrm{~h}$.

of freedom in the numerator and the denominator, respectively. The lack of a slope or curvature in the data excludes periods up to $\sim 3 \mathrm{~h}$.

In order to estimate the upper limit on the amplitude of any periodicity in the range of $4 \mathrm{~s}$ to $1.5 \mathrm{~h}$, we added artificial sinusoidal signals with various periods to the data and repeated the periodogram analysis. Amplitudes in excess of $\Delta R=0.006-0.009 \mathrm{mag}$ and $\Delta V_{R}=0.5-0.7 \%$ can be excluded, with the lowest sensitivity corresponding to periods close to the local maxima of the spectral window function. Hence, we find that any coherent periodicity present must have a fractional modulation of Stokes $V_{R}<0.7 \%$.

No significant photometric or polarimetric variability was detected. The periodogram in Fig. 2 is divided into two sections of which the left-hand one covers the more relevant longer periods that might be expected for a merger. The right-hand panel demonstrates that no periodicity is found down to $P_{\text {rot }}=4 \mathrm{~s}$, the break-up period of a $1.2 M_{\odot}$ white dwarf. For 6.950 trial periods each, in the photometric and in the polarimetric data, only a few frequency bins reach a significance above $3 \sigma$, entirely consistent with expectation for the detection of spurious lines in a wide frequency band. Folding of the data on the frequencies with $\sim 3 \sigma$ significance uncorrected for bandwidth demonstrates that none yields a sinusoidal modulation with an amplitude exceeding either $0.004 \mathrm{mag}$ in $I_{R}$ or $0.4 \%$ in $V_{R}$. Since the addition of an artificial sinusoidal signal to the data produces a peak in the periodogram with $F W H M \sim 3$ pixels, the number of independent trial
Table 1. Circular polarization in the red part of the spectrum obatined from spectropolarimetry and polarimetry in the Bessell $R$-band and in quasi $R$-bands. $\lambda_{\mathrm{c}}$ and $\Delta \lambda$ refer to the central wavelength and the width of the respective band.

\begin{tabular}{lccccc}
\hline Date & HJD & $\begin{array}{c}\lambda_{\mathrm{c}} \\
(\AA)\end{array}$ & $\begin{array}{c}\Delta \lambda \\
(\AA)\end{array}$ & $\begin{array}{c}V_{R} \\
(\%)\end{array}$ & Ref. \\
\hline 1977 Feb. 22 & 2443196.5 & 6250 & 1500 & $10.2 \pm 0.5$ & 1 \\
$\quad$ Feb. 23 & 2443197.5 & & & $10.4 \pm 0.5$ & 1 \\
1986 & & 6500 & 1300 & $10.0 \pm 0.7$ & 2 \\
1987 Feb. 23.9 & 2446850.4 & 7000 & 1000 & $8.2 \pm 1.0$ & 3 \\
1994 May 7 & 2449480.4 & 6550 & 1700 & $6.0 \pm 0.5$ & 4 \\
2000 Feb. 4 & 2451578.6 & 6550 & 1700 & $9.1 \pm 0.3$ & 5 \\
2000 Jul. 3/4 & 2451729.5 & 6100 & 800 & $9.5 \pm 0.3$ & 6 \\
\hline
\end{tabular}

References: (1) Liebert et al. (1978), (2) West (1989), (3) Robert \& Moffat (1989), (4) Schmidt et al. (1995), (5) This work, (6) Jordan \& Friedrich (2001).

periods within the total bandwith is reduced to $\sim 2.300$. Corrected for the bandwith, the Fisher-Snedecor critical value for a $3-\sigma$ detection of a periodic signal in the data then is $\Theta_{\text {cr }}=4.39$. We conclude that there is no evidence for periodic variation in both the photometric and the polarimetric variation.

The mean of our $R$-band circular polarization values is $V_{R}=9.1 \pm 0.3 \%$. We summarize this and other published polarization measurements in the red part of the spectrum (quasi $R$-bands) in Table 1 . The spectropolarimetric observations of Liebert et al. (1978) and Schmidt et al. (1995) have been averaged over pass bands as close as possible to 
the Kron-Cousins $R$-band. Since the circular polarization falls off from a maximum at $5750 \AA$ towards longer wavelengths (Schmidt et al. 1995, their Fig. 2), the values quoted in Table 1 are not strictly comparable. We have tried to account for this uncertainty in assigning the errors. The quoted circular polarizations are consistent with each other, except for the low value measured from the polarization spectrum of Schmidt et al. (1995). Taken at face value, the data in Table 1 suggest that there is a variation over the time span of 23 years with a period of this order. The sparcity of the data calls, however, for more extensive polarimetric monitoring with a single instrument, either broad band polarimetry or preferably spectropolarimetry as described by Jordan \& Friedrich (2001)

\section{Discussion and conclusion}

We have performed a sensitive search for short periodicities in the $R$-band flux and circular polarization of the highly magnetic white dwarf LP790-29 which was previously thought to have a rotational period $P_{\text {rot }}>100$ yrs (Schmidt \& Norsworthy 1991). We undertook this search because the sparse data available so far may have prevented the discovery of short periods.

Rapid rotation would be the signature of a white dwarf spun up in a cataclysmic variable of the AM Herculis type (Meyer \& Meyer-Hofmeister 1999), of the AM Canes Venaticorum type (Iben \& Tutukov 1991), or in a double degenerate merger (e.g. Segretain et al. 1997; Ferrario et al. 1997). A merger may rotate at the disruption limit. Segretain et al. argued, however, that the merger loses $90 \%$ of the initial angular momentum by a strong wind, yielding an initial rotational period $P_{\text {rot }} \sim 1 \mathrm{~min}$. At an effective temperature of about $8000 \mathrm{~K}$ (Liebert et al. 1978; Bues 1999) the cooling age of LP 790-29 is $t_{\text {cool }} \simeq 2 \times 10^{9}$ yrs (Anselowitz et al. 1999), but may be shorter if it originated from the merger of a cool white dwarf with its companion (Segretain et al. 1997). Hence, even a cool white dwarf might still be a fast rotator, although over the time the original rotational velocity may have been reduced by magnetic braking.

Our principal result is the absence of variability in LP 790-29 with periods between $4 \mathrm{~s}$ and about $3 \mathrm{~h}$ and amplitudes $\Delta R>0.009 \mathrm{mag}$ and $\Delta V_{R}>0.7 \%$. This includes the absence of photometric variability of the type one might expect in a short period binary. Hence, there is no positive evidence for fast rotation and no evidence for any of the above scenarios.

The only remaining possibilities which could mask rapid rotation in LP 790-29 are (i) the almost perfect alignment of the rotational and magnetic axes of an azimuthally symmetric field or (ii) a rotational axis oriented directly towards the observer, leading to rotational variability below our detection limit of the circular polarization.

Previous circular polarimetry excludes periods longer than $P_{\text {rot }} \sim 1 \mathrm{~h}$, although the limit $P_{\text {rot }}>100$ yrs set by
West (1989) and Schmidt \& Norsworthy (1991) may be premature in view of the low level of the 1994 circular polarization by Schmidt et al. (1995). Nevertheless, these results suggest that LP 790-29 is, in fact, an exceedingly slow rotator. It seems worthwhile to follow up the possibility of a period of about a quarter of a century (see also the paper by Jordan \& Friedrich 2001) by monitoring the level of circular polarization.

Acknowledgements. We thank Stefan Jordan, Boris T. Gänsicke, Frederic V. Hessman for valuable comments on the manuscript. This work was supported in part by BMBF/DLR grant 50 OR 99036.

\section{References}

Achilleos, N., Wickramasinghe, D. T., Liebert, J., Saffer, R. A., \& Grauer, A. D. 1992, ApJ, 396, 273

Anselowitz, T., Wasatonic, R., Metthews, K., Sion, E., \& McCook, G. P. 1999, PASP, 111, 702

Barstow, M. A., Jordan, S., O'Donoghue, D., et al. 1995, MNRAS, 277, 971

Berdyugin, A. V., \& Piirola, V. 1999, A\&A, 352, 619

Bues, I. 1999, ASP Conf. Ser., 169, 240

Burleigh, M. R., Jordan, S., \& Schweizer, W. 1999, ApJ, 510, L37

Ferrario, L., Vennes, S., Wickramasinghe, D. T., Bailey, J. A., \& Christian, D. J. 1997, MNRAS, 292, 205

Friedrich, S., \& Jordan, S. 2001, A\&A, 367, 577

Gänsicke, B. T. 2000, Rev. Mod. Astron., 13, 151

Heber, U., Napiwotzki, R., \& Reid, I. N. 1997, A\&A, 323, 819

Heyl, J. S. 2000, MNRAS, 317, 310

Howell, S., \& Ciardi, D. R. 2001, ApJ, 550, 57

Iben, I., Jr., \& Tutukov, A. V. 1991, ApJ, 370, 615

Jordan, S., \& Friedrich, S. 2001, A\&A, in press

Koester, D., Dreizler, S., Weidemann, V., \& Allard, N. F. 1998, A\&A, 338, 612

Liebert, J., Angel, J. R. P., Stockman, H. S., Spinrad, H., \& Beaver, E. A. 1977, ApJ, 214, 457

Liebert, J., Angel, J. R. P., Stockman, H. S., \& Beaver, E. A. 1978, ApJ, 225, 181

Meyer, F., \& Meyer-Hofmeister, E. 1999, A\&A, 346, L13

Robert, C., \& Moffat, A. F. J. 1989, ApJ, 343, 902

Schmidt, G. D., \& Norsworthy, J. E. 1991, ApJ, 366, 270

Schmidt, G. D., Bergeron, P., \& Fegley, B. 1995, ApJ, 443, 274

Schmidt, G. D., \& Smith, P. S. 1995, ApJ, 448, 305

Schmidt, G. D., Liebert, J., Harris, H. C., Dahn, C. C., \& Leggett, S. K. 1999, ApJ, 512, 916

Schwarzenberg-Czerny, A. 1989, MNRAS, 241, 153

Segretain, L., Chabrier, G., \& Mochkovitch, R. 1997, ApJ, 481, 355

West, S. C. 1989, ApJ, 345, 511

Wickramasinghe, D. T., \& Bessel, M. S. 1979, MNRAS, 188, 841

Wickramasinghe, D. T., \& Cropper, M. 1988, MNRAS, 235, 1451

Wickramasinghe, D. T., \& Ferrario, L. 2000, PASP, 112, 873 